\title{
Distinguishing modified gravity with just two tensorial degrees of freedom from general relativity: Black holes, cosmology, and matter coupling
}

\author{
Aya Iyonaga ${ }^{1, *}$ and Tsutomu Kobayashi ${ }^{1, \dagger}$ \\ ${ }^{1}$ Department of Physics, Rikkyo University, Toshima, Tokyo 171-8501, Japan
}

\begin{abstract}
We consider spatially covariant modified gravity in which the would-be scalar degree of freedom is made non-dynamical and hence there are just two tensorial degrees of freedom, i.e., the same number of dynamical degrees of freedom as in general relativity. Focusing on a class of such modified gravity theories characterized by three functions of time, we discuss how modified gravity with two tensorial degrees of freedom can be distinguished observationally or phenomenologically from general relativity. It is checked that the theory gives the same predictions as general relativity for weak gravitational fields and the propagation speed of gravitational waves. We also find that there is no modification to asymptotically flat black hole solutions. Due to a large degree of freedom to choose the time-dependent functions in the theory, the homogeneous and isotropic cosmological dynamics can be made close to or even identical to that of the $\Lambda$ CDM model. We investigate the behavior of cosmological perturbations in the long and short wavelength limits and show that in both limits the effects of modified gravity appear only through the modification of the background evolution. Finally, it is remarked that in the presence of a galileon field in the matter sector, the scalar degree of freedom is revived, ruining the essential feature of the theory.
\end{abstract}

\section{INTRODUCTION}

According to Lovelock's theorem [1, 2], the Einstein tensor plus a cosmological term is the only possible second-order Euler-Lagrange equation that is obtained from a diffeomorphism invariant action constructed from the metric tensor alone in four spacetime dimensions. Modifying gravity amounts to relaxing the postulates of the theorem. Abandoning full spacetime diffeomorphism invariance, one can for example consider modified gravity enjoying only three-dimensional spatial diffeomorphism invariance. Basically, this way of modifying gravity is equivalent to introducing a new dynamical scalar degree of freedom (DOF) that spontaneously breaks time diffeomorphism invariance. This is the underlying idea utilized in the construction of effective field theories for inflation and dark energy [3-6]. A general framework for scalartensor theories based on this idea has been developed in Refs. $[7,8]$.

An interesting twist is that a scalar DOF incorporated in such a framework is not necessarily dynamical and it is possible that modified gravity in which time diffeomorphism invariance is broken has only two tensorial degrees of freedom as general relativity (GR). The simplest example of such "scalarless" scalar-tensor theories is the cuscuton theory [9], in which the scalar field has an infinite propagation speed. An attempt to identify the cuscuton-like subclass within the Horndeski [10] and beyond Horndeski [11] theories is presented in Ref. [12]. Other modified gravity theories with two DOFs have been constructed in Refs. [13-17], a subclass of which is in fact equivalent to the cuscuton theory [18]. On the basis of the general framework of spatially covariant the-

\footnotetext{
* Email: iyonaga@rikkyo.ac.jp

$\dagger$ Email: tsutomu@rikkyo.ac.jp
}

ories of gravity [7, 8], Ref. [19] has derived through a rigorous Hamiltonian analysis the conditions under which there are only two DOFs and presented a particular action satisfying the conditions. The action is composed of two derivative terms built out of three-dimensional geometrical quantities as in GR, but depends on the lapse function in a nontrivial manner. A complementary perturbative approach to spatially covariant modified gravity without a scalar DOF has been proposed in Ref. [20].

The purpose of this paper is to explore phenomenological aspects of the theory of modified gravity with two tensorial DOFs proposed in Ref. [19] to see how the theory can be distinguished observationally from GR. We will see that it is quite difficult to find differences between the two theories in the weak gravity regime, gravitational wave propagation, black-hole spacetimes, and cosmology, when restricted to a certain subset of the theories of Ref. [19]. The theory of modified gravity we study in this paper is somewhat similar to the infrared limit of Hor̆ava gravity [21] in some respects, and we share the same motivation, e.g., as Ref. [22].

The structure of this paper is as follows. In the next section, we review modified gravity theories with two tensorial DOFs [19] which we study in this paper. In Sec. III, we identify the subclass of the theories that evades solar-system and gravitational-wave constraints. Then, we study black hole spacetimes in Sec. IV and cosmological aspects in Sec. V. In Sec. VI, a side remark concerning a coupling to a galileon field is given. Finally, we draw our conclusions in Sec. VII.

\section{SPATIALLY COVARIANT GRAVITY WITH TWO TENSORIAL DEGREES OF FREEDOM}

In this section, we introduce a class of spatially covariant modified gravity in which there are just two tensorial DOFs [19] and in particular there is no scalar DOF. 


\section{A. "Scalarless" scalar-tensor theories}

Let us begin with scalar-tensor theories with one scalar DOF, $\phi$, on top of two tensorial DOFs. In the unitary gauge where $\phi$ is homogeneous on constant time hypersurfaces, ghost-free scalar-tensor theories can in general be described by the action of the form $[7,8]$,

$$
S=\int \mathrm{d} t \mathrm{~d}^{3} x N \sqrt{\gamma} \mathcal{L}\left(N, \gamma_{i j}, K_{i j}, R_{i j}, D_{i} ; t\right),
$$

where $N$ is the lapse function, $\gamma_{i j}$ is the spatial metric, $K_{i j}$ is the extrinsic curvature of constant time hypersurfaces,

$$
K_{i j}=\frac{1}{2 N}\left(\partial_{t} \gamma_{i j}-D_{i} N_{j}-D_{j} N_{i}\right)
$$

with $N_{i}$ being the shift vector, $R_{i j}$ is the intrinsic curvature tensor, and $D_{i}$ is the covariant derivative with respect to $\gamma_{i j}$. The Horndeski action [10, 23, 24] can be recast in the above form in terms of these ArnowittDeser-Misner (ADM) variables. One may also include the velocity of the lapse function, $\partial_{t} N-N^{i} D_{i} N$, and explore a larger class of healthy scalar-tensor theories with three DOFs [25]. Although such a generalization leads to an interesting class of theories called degenerate higher-order scalar-tensor theories [26-28], we focus for simplicity on the action of the form (1).

Thus, in general, the action (1) describes scalar-tensor theories with three DOFs. However, in a particular subset of theories, the scalar mode obeys an elliptic equation and does not propagate. Consequently, such theories have only two tensorial DOFs as in GR, but the action is certainly different from that of GR. A particular example is given by [19]

$$
\begin{aligned}
S=\frac{1}{2} & \int \mathrm{d} t \mathrm{~d}^{3} x N \sqrt{\gamma}\left[\frac{\beta_{0} N}{\beta_{2}+N} K_{i j} K^{i j}\right. \\
& -\frac{\beta_{0}}{3}\left(\frac{2 N}{\beta_{1}+N}+\frac{N}{\beta_{2}+N}\right) K^{2} \\
& \left.+\alpha_{1}+\alpha_{2} R+\frac{1}{N}\left(\alpha_{3}+\alpha_{4} R\right)\right]
\end{aligned}
$$

where $\beta_{A}$ and $\alpha_{A}$ are functions of $t$. A rigorous Hamiltonian analysis has shown that this theory indeed has only two tensorial DOFs [19]. ${ }^{1}$ The cuscuton theory [9] is reproduced by setting $\beta_{0}=\alpha_{2}=1$ and $\beta_{1}=\beta_{2}=\alpha_{4}=0$, with $\alpha_{3}$ coming from the cuscuton term $\sigma(\phi) \sqrt{-\partial_{\mu} \phi \partial^{\mu} \phi}$, while the extended cuscuton theories [12, 29] correspond to the case with $\beta_{1}=\beta_{2}$. The covariant expression for the action (3) in terms of a Stückelberg scalar field is

\footnotetext{
1 The case with $\beta_{0}=1$ is presented in Ref. [19], but it is clear from the footnote of Ref. [19] that one can include this time-dependent coefficient.
}

given in Appendix B. It is instructive to rewrite the kinetic part of the Lagrangian as

$$
\frac{\beta_{0}}{2}\left(\frac{N}{\beta_{2}+N} \widetilde{K}_{i j} \widetilde{K}^{i j}-\frac{2}{3} \frac{N}{\beta_{1}+N} K^{2}\right),
$$

where $\widetilde{K}_{i j}:=K_{i j}-(1 / 3) K \gamma_{i j}$ is the traceless part of the extrinsic curvature. This expression shows the roles of the functions $\beta_{1}$ and $\beta_{2}$ more clearly. In the following we will explore various aspects of the gravitational theory with the action (3).

Before proceeding let us comment on other examples of "scalarless" scalar-tensor theories with two DOFs. Under the assumption that the Lagrangian is linear in the lapse function (i.e., $\mathcal{L}$ in Eq. (1) is independent of $N$ ), the conditions for a theory to have only two tensorial DOFs have been addressed in Ref. [13]. References [14-16] obtained gravitational theories with two DOFs via canonical transformations from GR in the Hamiltonian formulation. In the context of the recent claim concerning the $D \rightarrow 4$ limit of $D$-dimensional Gauss-Bonnet gravity [30], a consistent theory with two DOFs has been proposed [17] that avoids the propagation of the scalar mode present in the previous derivations [31-35]. Symmetries that prohibit the scalar mode from propagating have been discussed in Ref. [36]. See Refs. [37-39] for further developments in "scalarless" modified gravity and Refs. [40-42] for the application to cosmology.

\section{B. Quasi-Einstein frame}

A field redefinition of a metric given by a disformal transformation [43] retains the number of propagating DOFs as long as the transformation is invertible. In terms of the ADM variables, it is expressed as

$$
N \rightarrow \mathcal{N}(t, N), \quad N^{i} \rightarrow N^{i}, \quad \gamma_{i j} \rightarrow \mathcal{A}(t, N) \gamma_{i j}
$$

Let us consider a special case of a disformal transformation,

$$
N \rightarrow b_{0}(t) N+b_{1}(t), \quad N^{i} \rightarrow N^{i}, \quad \gamma_{i j} \rightarrow \gamma_{i j}
$$

Under the transformation (6), the form of the action (3) is invariant, with $\beta_{A}(t)$ and $\alpha_{A}(t)$ being transformed as

$$
\begin{aligned}
& \beta_{0} \rightarrow \beta_{0} / b_{0}, \quad \beta_{1,2} \rightarrow\left(\beta_{1,2}+b_{1}\right) / b_{0} \\
& \alpha_{1,2} \rightarrow b_{0} \alpha_{1,2}, \quad \alpha_{3,4} \rightarrow \alpha_{3,4}+b_{1} \alpha_{1,2} .
\end{aligned}
$$

Therefore, via the field redefinition (6) one can always set $\beta_{0}=1$ and $\beta_{2}=0$. Since the coefficient of $K_{i j} K^{i j}$ is now standard, we dub this frame as the quasi-Einstein 
frame. This motivates us to study the action of the form ${ }^{2}$

$$
\begin{gathered}
S=\frac{1}{2} \int \mathrm{d} t \mathrm{~d}^{3} x N \sqrt{\gamma}\left[K_{i j} K^{i j}-\frac{1}{3}\left(\frac{2 N}{\beta+N}+1\right) K^{2}\right. \\
\left.+\alpha_{1}+\alpha_{2} R+\frac{1}{N}\left(\alpha_{3}+\alpha_{4} R\right)\right]
\end{gathered}
$$

where $\beta:=\beta_{1}$, as the case of particular interest. General relativity with a cosmological constant is reproduced by setting $\beta=\alpha_{3}=\alpha_{4}=0, \alpha_{1}=$ const, and $\alpha_{2}=1$. Theories with $\beta \neq 0$ are not included in the (extended) cuscuton family, and hence the impacts of $\beta$ have not been investigated so far in the literature.

Note that a subtlety arises when matter is present. In this paper we assume that matter is minimally coupled to gravity in this quasi-Einstein frame.

Here we should point out the similarity and difference between the action (8) and that of the infrared limit of nonprojectable Hořava gravity [21, 44, 45],

$$
\begin{aligned}
S=\frac{1}{2} \int \mathrm{d} t \mathrm{~d}^{3} x N \sqrt{\gamma}\left(K_{i j} K^{i j}-\lambda K^{2}\right. \\
\left.+\alpha_{1}+\alpha_{2} R+\eta a_{i} a^{i}\right)
\end{aligned}
$$

where $a_{i}:=D_{i} \ln N$ and now all the coefficients are constants. The same action is also obtained in the context of Einstein-aether theory [46] if the aether field is restricted to be hypersurface orthogonal [47, 48]. While being mostly similar, the essential difference can be perceived in the structure of the coefficient of $K^{2}$. In the case of $\eta=0$, nonprojectable Hořava gravity has odd dimensionality of the phase space at each spacetime point and hence is inconsistent $[49,50]$. By contrast, the action (8) is free of such a trouble thanks to the particular structure of the coefficient of $K^{2}$.

\section{SOLAR-SYSTEM TESTS AND GRAVITATIONAL WAVES}

Let us study gravitational potentials produced by nonrelativistic matter and the propagation of gravitational waves in the theory described by the action (8). We assume that $\alpha_{1}, \alpha_{3} \sim H_{0}^{2}$, where $H_{0}$ is the Hubble scale, so that they are only relevant to the large-scale cosmological dynamics. We also assume that $\alpha_{2}$ and $\alpha_{4}$ could vary only on cosmological time scales, because otherwise nearly static gravitational potentials would not be possible. We can then consider scalar and tensor perturbations on a Minkowski background, using the approximation $\alpha_{1}, \alpha_{3} \ll \partial_{t}^{2}, \Delta:=\delta_{i j} \partial_{i} \partial_{j}$.

\footnotetext{
2 As will be clear shortly, we use units in which Newton's constant, $G_{N}$, is equal to $\left(8 \pi \alpha_{2}\right)^{-1}$, and we will be interested mainly in the case with $\alpha_{2}=1$.
}

Static scalar perturbations of the ADM variables are given by

$$
N=1+\Phi(\vec{x}), \quad N_{i}=\partial_{i} \chi(\vec{x}), \quad \gamma_{i j}=[1-2 \Psi(\vec{x})] \delta_{i j}
$$

The quadratic action reads

$$
\begin{gathered}
S=\int \mathrm{d} t \mathrm{~d}^{3} x\left[-\left(\alpha_{2}+\alpha_{4}\right) \Psi \Delta \Psi+2 \alpha_{2} \Phi \Delta \Psi\right. \\
\left.+\frac{\beta}{3(1+\beta)}(\Delta \chi)^{2}-\Phi \rho(\vec{x})\right]
\end{gathered}
$$

where $\rho(\vec{x})$ is the matter energy density and this matter is assumed to be minimally coupled to gravity. Note that in deriving Eq. (11) we actually assumed that $\beta \Delta \gg H_{0}^{2}$. The equations of motion are then solved to give

$$
\Delta \Phi=\left(1+\frac{\alpha_{4}}{\alpha_{2}}\right) \frac{\rho}{2 \alpha_{2}}, \quad \Delta \Psi=\frac{\rho}{2 \alpha_{2}}, \quad \Delta \chi=0
$$

This result implies that

$$
\alpha_{2} \simeq \text { const } \text { and } \quad \alpha_{4} \simeq 0
$$

are required in order to evade solar-system constraints (light bending, the Shapiro time delay, and the time variation of Newton's constant). ${ }^{3}$

Tensor perturbations are given by

$$
N=1, \quad N_{i}=0, \quad \gamma_{i j}=\delta_{i j}+h_{i j}(t, \vec{x})
$$

for which the quadratic action is

$$
S=\frac{1}{8} \int \mathrm{d} t \mathrm{~d}^{3} x\left[\left(\partial_{t} h_{i j}\right)^{2}+\left(\alpha_{2}+\alpha_{4}\right) h_{i j} \Delta h_{i j}\right] .
$$

The observation of GW170817 and GRB 170817A put a tight bound on the speed of gravitational waves, $c_{\mathrm{GW}} \simeq$ $1[52,53]$. It is therefore required that

$$
\alpha_{2}+\alpha_{4} \simeq 1
$$

Summarizing these results, we identify a phenomenologically interesting class characterized by

$$
\alpha_{2}=1, \quad \alpha_{4}=0
$$

which evades solar-system and gravitational-wave constraints. Note that in these noncosmological phenomena we do not see any effects of nonvanishing $\beta$.

\footnotetext{
3 According to the current limits on the time variation of Newton's constant, $\left|\dot{\alpha}_{2} / \alpha_{2}\right|$ must be much smaller than $H_{0}[51]$.
} 


\section{BLACK HOLE SOLUTIONS}

In this section, we study black hole solutions in the theory defined by the action (8). On the basis of the discussion in the previous section, we are interested in the phenomenologically viable case with $\alpha_{2}=1$ and $\alpha_{4}=0$. Black holes under consideration are supposed to be much smaller than the size of the cosmological horizon. Therefore, we are mostly interested in asymptotically flat black holes obtained ignoring $\alpha_{1}$ and $\alpha_{3}$. (These two functions are assumed to be characterized by the cosmological horizon scale.) The remaining unfixed function, $\beta$, is assumed to be constant in this section, because we seek for stationary black hole solutions. Our focus is therefore on whether the effects of $\beta$ can be seen or not in black hole solutions. Nevertheless, having said that, we allow $\alpha_{1}$ to be a nonvanishing constant and $\alpha_{2}$ to be a constant different from 1 in the following discussion, because relaxing these assumptions does not hinder us from presenting an analytic solution below. To sum up, in this section we have three constant parameters, $\beta, \alpha_{1}$, and $\alpha_{2}$, though we are mostly interested in the case with $\alpha_{1}=0$ and $\alpha_{2}=1$.

\section{A. Static and spherically symmetric solution}

The ADM variables for static and spherically symmetric solutions are taken to be

$$
\begin{aligned}
& N=N(r), \quad N_{i} \mathrm{~d} x^{i}=B(r) F(r) \mathrm{d} r, \\
& \gamma_{i j} \mathrm{~d} x^{i} \mathrm{~d} x^{j}=F^{2}(r) \mathrm{d} r^{2}+r^{2} \mathrm{~d} \Omega^{2},
\end{aligned}
$$

where $\mathrm{d} \Omega^{2}=\mathrm{d} \theta^{2}+\sin ^{2} \theta \mathrm{d} \varphi^{2}$. Since the action is only invariant under spatial diffeomorphisms, at this stage we do not make a temporal coordinate transformation, $t \rightarrow$ $T(t, r)$, to remove $B$. Such a coordinate transformation would result in an inhomogeneous configuration of the nondynamical scalar field.

Substituting the ADM variables (18) to the action and varying it with respect to $N, B$, and $F$, we obtain

$$
\begin{aligned}
& \alpha_{1}+\frac{2 \alpha_{2}}{r^{2}}\left[\left(\frac{F^{2}-1}{F^{2}}\right) r\right]^{\prime}+\frac{2\left(r B^{2}\right)^{\prime}}{r^{2}(\beta+N)^{2} F^{2}} \\
& -\frac{2 r^{2}}{3} \frac{\beta(\beta+2 N)}{N^{2}(\beta+N)^{2} F^{2}}\left[(B / r)^{\prime}\right]^{2}=0 \\
& \frac{r^{2} B}{(\beta+N)^{2} F^{2}}[(\beta+N) F]^{\prime}+\frac{\beta}{3}\left[\frac{r^{4}(B / r)^{\prime}}{N(\beta+N) F}\right]^{\prime}=0 \\
& \alpha_{1} \frac{N}{F}+\frac{2 \alpha_{2}}{r^{2}} \frac{N}{F}\left(\frac{F^{2}-1}{F^{2}}-\frac{2 r N^{\prime}}{N F^{2}}\right) \\
& +\frac{2\left(r B^{2}\right)^{\prime}}{r^{2} N F^{3}}-\frac{2 \beta}{3 r^{4} N(\beta+N) F^{3}}\left[\left(r^{2} B\right)^{\prime}\right]^{2}=0
\end{aligned}
$$

where a dash denotes differentiation with respect to $r$. The system is of second order for $B$ (provided that $\beta \neq 0$ ) and of first order for $N$ and $F$.
Since it is difficult to obtain a general solution to Eqs. (19)-(21), we start with finding a particular solution. One can easily verify that Eqs. (19)-(21) admit the following solution:

$$
N=N_{0} \sqrt{f(r)}, \quad F=\frac{1}{\sqrt{f(r)}}, \quad B=\frac{N_{0} b_{0}}{r^{2}},
$$

where

$$
f(r):=1+\frac{\alpha_{1}}{6 \alpha_{2}} r^{2}-\frac{\mu_{0}}{r}+\frac{b_{0}^{2}}{\alpha_{2} r^{4}},
$$

and $N_{0}, \mu_{0}$, and $b_{0}$ are integration constants. Here, $N_{0}$ is physically less important because it may be set to 1 by rescaling the unit of $t$.

To see the spacetime structure of this solution more clearly, we note that the four-dimensional metric can be written as

$$
\begin{aligned}
\mathrm{d} s^{2}= & -\left(N^{2}-B^{2}\right)\left(\mathrm{d} t-\frac{B F}{N^{2}-B^{2}} \mathrm{~d} r\right)^{2} \\
& +\frac{N^{2} F^{2}}{N^{2}-B^{2}} \mathrm{~d} r^{2}+r^{2} \mathrm{~d} \Omega^{2}
\end{aligned}
$$

where

$$
\begin{aligned}
N^{2}-B^{2} & =N_{0}^{2}\left(1+\frac{\alpha_{1}}{6 \alpha_{2}} r^{2}-\frac{\mu_{0}}{r}+\frac{1-\alpha_{2}}{\alpha_{2}} \frac{b_{0}^{2}}{r^{4}}\right), \\
N F & =N_{0} .
\end{aligned}
$$

Thus, introducing a new time coordinate defined by $\mathrm{d} T=N_{0}\left\{\mathrm{~d} t-\left[B F /\left(N^{2}-B^{2}\right)\right] \mathrm{d} r\right\}$, one can write the metric in a diagonal form at the expense of a homogeneous configuration of the nondynamical scalar field (see Appendix B). It is now obvious that in the phenomenologically interesting case of $\alpha_{2}=1$, the solution (22) describes Schwarzschild-(anti-)de Sitter spacetime. Importantly, we see no $\beta$-dependence in this solution. Note that inside the black hole horizon there is a location at which $N=0$. If $\alpha_{1}<0$, such a location also exists outside the cosmological horizon. These are called the universal horizon, which has been studied in Einstein-aether theory and Hořava gravity [54, 55].

Aside from the cosmological constant term, essentially the same expression for a black hole solution has been obtained in Einstein-aether theory [56]. This is not surprising because the solution (22) satisfies the maximal slicing condition, $K=-\left(r^{2} B\right)^{\prime} / r^{2} N F=0$, while the crucial difference between the two theories is found in the coefficient of $K^{2}$ in the action. The maximal slicing condition is the very reason why there is no $\beta$-dependence in the solution. Note that a similar solution was found in a different modified gravity theory with two tensorial DOFs [57].

\section{B. Static perturbations}

We have thus obtained a particular solution (22), but at this stage it is not clear whether or not there exist 
other black hole solutions with the appropriate asymptotic behavior. Since $B$ obeys the second-order differential equations, one might rather expect that there should be another integration constant in addition to $N_{0}, \mu_{0}$, and $b_{0}$. To address this point, let us consider a slightly deformed solution,

$$
\begin{aligned}
& N=N_{0} \sqrt{f+h_{0}(r)}, \quad F=\frac{1}{\sqrt{f+h_{1}(r)}}, \\
& B=N_{0}\left[\frac{b_{0}}{r^{2}}+h_{2}(r)\right]
\end{aligned}
$$

where $h_{1}, h_{2}$, and $h_{3}$ are to be treated as small perturbations.

Equations (19) and (21) reduce, respectively, to

$$
\begin{aligned}
& f\left[\frac{2 b_{0}}{\alpha_{2}}\left(\frac{h_{2}}{r}\right)^{\prime}-\left(r h_{1}\right)^{\prime}\right]+\frac{3 b_{0}^{2}}{\alpha_{2} r^{4}}\left(h_{0}-h_{1}\right)=0 \\
& f\left[\frac{2 b_{0}}{\alpha_{2}}\left(\frac{h_{2}}{r}\right)^{\prime}-\left(r h_{0}\right)^{\prime}\right]+\left(1+\frac{\alpha_{1}}{2 \alpha_{2}} r^{2}\right)\left(h_{0}-h_{1}\right)=0 .
\end{aligned}
$$

Eliminating $h_{2}$ from these two equations, we obtain

$$
f\left(h_{0}-h_{1}\right)^{\prime}-f^{\prime}\left(h_{0}-h_{1}\right)=0,
$$

which can be integrated to give

$$
h_{0}-h_{1}=C_{1} f
$$

where $C_{1}$ is an integration constant. This integration constant can be absorbed into a redefinition of $N_{0}$. Substituting this to Eq. (28), we have

$$
\begin{aligned}
& h_{0}=-\frac{\mu_{1}}{r}+C_{1}\left(f-\frac{b_{0}^{2}}{\alpha_{2} r^{4}}\right)+\frac{2 b_{0}}{\alpha_{2}} \frac{h_{2}}{r^{2}}, \\
& h_{1}=-\frac{\mu_{1}}{r}-\frac{C_{1} b_{0}^{2}}{\alpha_{2} r^{4}}+\frac{2 b_{0}}{\alpha_{2}} \frac{h_{2}}{r^{2}}
\end{aligned}
$$

where $\mu_{1}$ is an integration constant. This integration constant simply corresponds to a constant shift of the mass parameter $\mu_{0}$. Finally, substituting all these results to Eq. (20), we arrive at

$$
\begin{aligned}
& \left(\beta+N_{0} \sqrt{f}\right)\left(h_{2}^{\prime \prime}+\frac{2}{r} h_{2}^{\prime}-\frac{2}{r^{2}} h_{2}\right) \\
& -N_{0}(\sqrt{f})^{\prime}\left(h_{2}^{\prime}+\frac{2}{r} h_{2}\right)=0,
\end{aligned}
$$

where we assumed $\beta \neq 0$. This equation can be integrated to give

$$
h_{2}=\frac{b_{1}}{r^{2}}+\frac{\epsilon_{1}}{r^{2}} \int^{r} r^{2}\left(\beta+N_{0} \sqrt{f}\right) \mathrm{d} r,
$$

where $b_{1}$ and $\epsilon_{1}$ are integration constants. The former integration constant corresponds to a constant shift of $b_{0}$, while the latter cannot be absorbed into a shift of

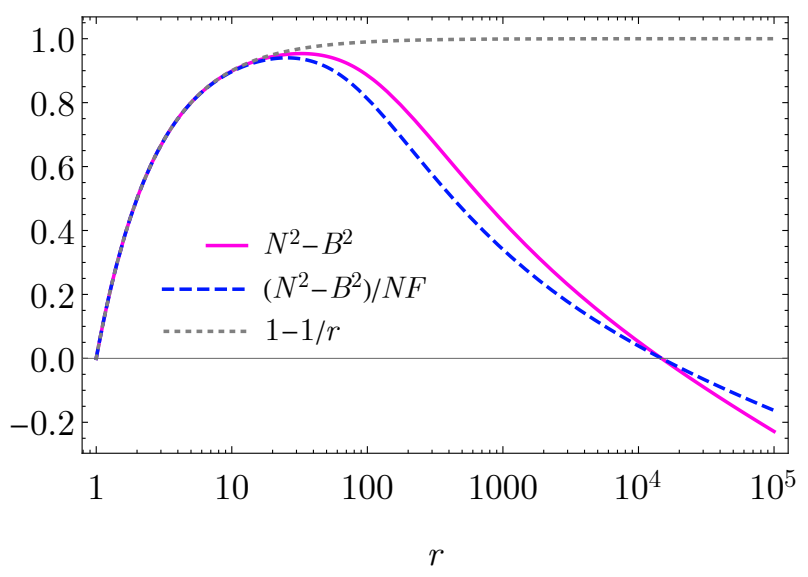

FIG. 1. Numerical solution for $r_{h}=1, N_{h}=0.1$, and $B_{1}=$ $-1.9 N_{h}$. The theory parameters are given by $\alpha_{1}=0, \alpha_{2}=1$, and $\beta=0.2$.



FIG. 2. Numerical solution for $r_{h}=1, N_{h}=0.1$, and $B_{1}=$ $-3 N_{h}$. The theory parameters are given by $\alpha_{1}=0, \alpha_{2}=1$, and $\beta=-0.3$.

the integration constants of the solution (22). Let us now focus specifically on the case of a zero cosmological constant, $\alpha_{1}=0$, where the solution is asymptotically flat at zeroth order. In this case, we have

$$
h_{2} \sim \frac{\epsilon_{1}}{3}\left(\beta+N_{0}\right) r
$$

for large $r$, which would invalidate the perturbative approximation unless $\epsilon_{1}=0$. Therefore, no asymptotically flat solution can be obtained by a small deformation of the solution (22). Our result agrees with that of the decoupling limit analysis of the khrononmetric theory [58].

\section{Numerical solutions}

We perform a numerical analysis that is complementary to and can go beyond the perturbative study in the 
previous subsection.

In the vicinity of the horizon, $r=r_{h}$, the ADM variables can be expanded as

$$
\begin{aligned}
& N=N_{h}+N_{1}\left(r-r_{h}\right)+\cdots, \\
& F=\frac{1}{N_{h}}+F_{1}\left(r-r_{h}\right)+\cdots, \\
& B=N_{h}+B_{1}\left(r-r_{h}\right)+\frac{B_{2}}{2}\left(r-r_{h}\right)^{2}+\cdots
\end{aligned}
$$

Here, one may set $F\left(r_{h}\right)=1 / N_{h}$ without loss of generality since doing so corresponds to fixing the scale of time. For given $\left(r_{h}, N_{h}, B_{1}\right)$, one can numerically integrate Eqs. (19)-(21) from the horizon outwards to determine the profile of $N, F$, and $B$. We thus try to find asymptotically flat solutions that are not described as a perturbation of the Schwarzschild spacetime foliated by maximal slices.

We consider again the case without the cosmological constant, $\alpha_{1}=0$. For

$$
B_{1}=-\frac{2 N_{h}}{r_{h}},
$$

one has the asymptotically flat solution given by Eq. (22) (with $\alpha_{1}=0$ ). As far as we have investigated numerically, no asymptotically flat solutions have been found if the condition (40) is not satisfied. ${ }^{4}$ Typical examples obtained in the case of detuned conditions are given in Figs. 1 and 2. The large $r$ behavior of these numerical solutions is also different from that of asymptotically de Sitter solutions.

Our numerical analysis thus supports the conclusion that asymptotically flat, spherically symmetric black holes in the theory defined by the action (8) with $\beta=$ const, $\alpha_{2}=1$, and $\alpha_{1}=\alpha_{3}=\alpha_{4}=0$ are indistinguishable from those in GR.

\section{Slowly rotating solution}

Let us now discuss slowly rotating black hole solutions (see Refs. [59-61] for rotating black holes in Einsteinaether theory and Hořava gravity). To first order in the black hole spin, we write $[62,63]$

$$
\delta N=0, \quad \delta N_{i} \mathrm{~d} x^{i}=-r^{2} \sin ^{2} \theta \omega(r, \theta) \mathrm{d} \varphi, \quad \delta \gamma_{i j}=0
$$

The $(r \varphi)$ component of the evolution equations reads

$$
\partial_{\theta}\left(\sin ^{3} \theta \partial_{\theta} \omega\right)=0
$$

\footnotetext{
4 We have not looked for asymptotically flat solutions in the case of $\alpha_{1} \neq 0$.
}

which, together with the regularity at the pole, yields $\omega=\omega(r)$. Then, the $\varphi$ component of the momentum constraints reduces to

$$
\left(\frac{r^{4} \omega^{\prime}}{N F}\right)^{\prime}=0
$$

This can be integrated to give

$$
\omega(r)=-\frac{3 J}{4 \pi} \int^{r} \frac{N F}{r^{4}} \mathrm{~d} r+\omega_{0},
$$

where $J$ and $\omega_{0}$ are integration constants. Upon using $N F=1$ we arrive at $\omega=J / 4 \pi r^{3}+\omega_{0}$. (For simplicity, here we have set $N_{0}=1$ without loss of generality.) Noting that the time coordinate $t$ that we are using is nonstandard, we perform a coordinate transformation

$$
\varphi \rightarrow \varphi+\int^{r} \frac{B F}{N^{2}-B^{2}}\left(\omega-\omega_{0}\right) \mathrm{d} r+\omega_{0} t .
$$

The four-dimensional metric then becomes

$$
\begin{aligned}
\mathrm{d} s^{2}= & -\left(N^{2}-B^{2}\right) \mathrm{d} T^{2}+\frac{\mathrm{d} r^{2}}{N^{2}-B^{2}}+r^{2} \mathrm{~d} \Omega^{2} \\
& -\frac{J \sin ^{2} \theta}{2 \pi r} \mathrm{~d} T \mathrm{~d} \varphi
\end{aligned}
$$

which is nothing but the Kerr metric in GR under the slow-rotation approximation. Thus, we conclude that no effect of $\beta$ can be seen in slowly rotating black holes.

\section{COSMOLOGY}

Our next step is the analysis of cosmology. In this section, we will mainly consider the phenomenologically most interesting case with the time-dependent functions

$$
\begin{aligned}
& \beta_{0}=1, \quad \beta_{1}=\beta(t), \quad \beta_{2}=0, \\
& \alpha_{1}=\alpha_{1}(t), \quad \alpha_{2}=1, \quad \alpha_{3}=\alpha_{3}(t), \quad \alpha_{4}=0 .
\end{aligned}
$$

However, when deriving a quadratic action for cosmological perturbations, we will work in the general action (3) where no assumptions are made on these functions, because the general form of the quadratic action will be used for another purpose in the next section.

To investigate cosmology, we add a (irrotational, barotropic) perfect fluid which is minimally coupled to gravity. Such a fluid component can be mimicked by a scalar field whose action is given by

$$
S_{\text {mat }}=\int \mathrm{d}^{4} x \sqrt{-g} P(Y), \quad Y:=-\frac{1}{2}(\partial \varphi)^{2} .
$$

The matter energy-momentum tensor reads

$$
T_{\mu \nu}=(\rho+P) u_{\mu} u_{\nu}+P g_{\mu \nu}
$$

where the energy density and the four-velocity are given respectively by

$$
\rho=2 Y P_{, Y}-P, \quad u_{\mu}=-\frac{\nabla_{\mu} \varphi}{\sqrt{2 Y}} .
$$


The equation of motion for $\varphi$ is equivalent to the conservation law, $\nabla_{\mu} T_{\nu}^{\mu}=0$. Here, $\nabla_{\mu}$ stands for the usual four-dimensional covariant derivative. The sound speed of the fluid is given by

$$
c_{s}^{2}=\frac{P_{, Y}}{P_{, Y}+2 Y P_{, Y Y}},
$$

where the right hand side is evaluated at the homogeneous background. For $P \propto Y^{(1+w) / 2 w}$ with $w=$ const, we have $c_{s}^{2}=P / \rho=w$.

\section{A. Homogeneous and isotropic background}

The total action is now composed of the gravity sector (8) and the action for a cosmological fluid (48).

The ADM variables for a homogeneous and isotropic universe are given by

$$
N=\bar{N}(t), \quad N_{i}=0, \quad \gamma_{i j}=a^{2}(t) \delta_{i j} .
$$

Since time reparametrization symmetry is spontaneously broken, one cannot put $\bar{N}=1$ in general, but, as we will show below, the lapse function is determined from the equations of motion. From the Hamiltonian constraint and the evolution equations, we obtain

$$
\begin{aligned}
& \frac{3 H^{2}}{(\beta / \bar{N}+1)^{2}}+\frac{\alpha_{1}}{2}=\rho \\
& -\frac{3 H^{2}}{\beta / \bar{N}+1}-\frac{2}{\bar{N}} \frac{\mathrm{d}}{\mathrm{d} t}\left(\frac{H}{\beta / \bar{N}+1}\right)-\frac{1}{2}\left(\alpha_{1}+\frac{\alpha_{3}}{\bar{N}}\right)=P
\end{aligned}
$$

where $H:=\bar{N}^{-1} \mathrm{~d} \ln a / \mathrm{d} t$ is the Hubble parameter. (As far as the spatially flat model is concerned, the coefficients of $R$, i.e., $\alpha_{2}$ and $\alpha_{4}$, do not appear anyway in the equations for a homogeneous universe.) The conservation law reads

$$
\bar{N}^{-1} \dot{\rho}+3 H(\rho+P)=0,
$$

where a dot stands for differentiation with respect to $t$. Given the equation of state, this equation can be integrated to give $\rho$ and $P$ as a function of $a$ : $\rho=\rho(a)$, $P=P(a)$. Note that Eq. (55) is not an automatic consequence of Eqs. (53) and (54). Rather, substitution of Eqs. (53) and (54) into Eq. (55) yields

$$
\begin{aligned}
& \dot{\alpha}_{1}-3 H \alpha_{3}-\frac{6 \beta H}{\beta / \bar{N}+1}\left[\frac{3 H^{2}}{\beta / \bar{N}+1}+\frac{2}{\bar{N}} \frac{\mathrm{d}}{\mathrm{d} t}\left(\frac{H}{\beta / \bar{N}+1}\right)\right] \\
& =0 .
\end{aligned}
$$

Using Eqs. (53) and (54) again, one can write this equation as the constraint among the time-dependent functions,

$$
\dot{\alpha}_{1}-\sqrt{3}\left(\rho-\frac{\alpha_{1}}{2}\right)^{1 / 2}\left[\alpha_{3}-\left(\alpha_{1}+2 P\right) \beta\right]=0 .
$$

In the covariant formulation presented in Appendix B, this follows from the equation of motion for a Stückelberg scalar field (see Eq. (B17)). In the ADM formulation of usual scalar-tensor theories with two tensorial and one scalar DOFs, the equation corresponding to Eq. (57) reduces to a first-order differential equation for the lapse function, which can be used to determine $\bar{N}=\bar{N}(t)$. In contrast, in the present case Eq. (57) does not contain $\bar{N}$, which is the crucial point in "scalarless" theories. Given that $\rho$ and $P$ are now expressed in terms of $a$, Eq. (57) instead allows us to write $a$ in terms of $t$. Finally, substituting $a=a(t)$ into Eq. (53), one can determine the lapse function $\bar{N}$ as a function of $t$.

Let us now present an example. Suppose that the universe is filled with a fluid with a constant equation of state parameter, $w=P / \rho$, and the time-dependent functions are given by

$$
\begin{aligned}
\beta & =\text { const }, \quad \alpha_{2}=1, \quad \alpha_{4}=0 \\
\alpha_{1}= & 6 h_{0}^{2}\left[\frac{1}{\xi}-\frac{1}{(1+\beta)^{2}}\right] \operatorname{coth}^{2}\left[\frac{3}{2}(1+w) h_{0} t\right]-\frac{6 h_{0}^{2}}{\xi} \\
\alpha_{3}= & 6 h_{0}^{2}\left[\frac{1+w(1+\beta)}{(1+\beta)^{2}}-\frac{1+w}{\xi}\right] \operatorname{csch}^{2}\left[\frac{3}{2}(1+w) h_{0} t\right] \\
& -\frac{6 \beta h_{0}^{2}}{(1+\beta)^{2}},
\end{aligned}
$$

where $\xi$ and $h_{0}$ are constant parameters. This example admits the following solution:

$$
\bar{N}=1, \quad a^{3(1+w)} \propto \sinh ^{2}\left[\frac{3}{2}(1+w) h_{0} t\right] .
$$

Noting that $\alpha_{1}$ can be written as

$$
\alpha_{1}=6\left[\frac{1}{\xi}-\frac{1}{(1+\beta)^{2}}\right] H^{2}-6 \frac{h_{0}^{2}}{\xi},
$$

we see that Eqs. (53) and (54) read

$$
\begin{aligned}
& 3 H^{2}=\xi \rho+3 h_{0}^{2}, \\
& -2 \dot{H}=\xi(\rho+P) .
\end{aligned}
$$

This shows that the cosmological gravitational constant differs from Newton's constant $G_{N}$ by the factor $\xi$ :

$$
\frac{G_{\mathrm{Cos}}}{G_{N}}=\xi
$$

(Recall that we are working in units where $8 \pi G_{N}=1$ (= $\left.1 / \alpha_{2}\right)$.) Aside from this modification, the background evolution obeys the standard Friedmann equations in the presence of a cosmological constant. A similar situation occurs in Einstein-aether theory and Hořava gravity, where a mild bound on the difference between $G_{\mathrm{cos}}$ and $G_{N}$ has been obtained from the measurement of the primordial abundance of $\mathrm{He}^{4}$ as $|\xi-1| \lesssim 0.1$ [44, 64]. In our example, $\xi$ is a free parameter so that one may set $\xi=1$, 
leading to the background evolution that is completely indistinguishable from that in GR.

In the above example, $\alpha_{1}$ and $\alpha_{3}$ track the cosmic evolution as $\alpha_{1}, \alpha_{3} \sim H^{2}$. We present another example in which $\alpha_{1}$ and $\alpha_{3}$ instead remain as small as $H_{0}^{2}$ and simply behave as a dark energy component with a constant equation of state parameter $w_{\mathrm{DE}}$. In the case where the matter component is a pressureless fluid, the model is given by

$$
\begin{aligned}
\beta & =\text { const, } \quad \alpha_{2}=1, \quad \alpha_{4}=0, \\
\alpha_{1} & =-2 \rho_{\mathrm{DE}}(t), \\
\alpha_{3} & =2\left[1+w_{\mathrm{DE}}(1+\beta)\right] \rho_{\mathrm{DE}}(t), \\
\rho_{\mathrm{DE}} & \propto A^{-3\left(1+w_{\mathrm{DE}}\right)},
\end{aligned}
$$

where $A$ and $t$ are related via

$$
\begin{aligned}
t= & \frac{2 \sqrt{1+r_{0}}}{3 H_{0}} A^{3 / 2} \\
& \times{ }_{2} F_{1}\left(1 / 2,-1 / 2 w_{\mathrm{DE}}, 1-1 / 2 w_{\mathrm{DE}},-r_{0} A^{-3 w_{\mathrm{DE}}}\right) .
\end{aligned}
$$

Here, ${ }_{2} F_{1}$ is the hypergeometric function and $r_{0}:=$ $\rho_{\mathrm{DE}} /\left.\rho\right|_{a=1}$ is the present value of the ratio between the dark energy density and the matter energy density. One can check that the solution is given by $\bar{N}=1$ and $a=A(t)$. This example yields the background evolution subject to

$$
\begin{aligned}
3 H^{2} & =(1+\beta)^{2}\left(\rho+\rho_{\mathrm{DE}}\right), \\
-2 \dot{H} & =(1+\beta)^{2}\left[\rho+\left(1+w_{\mathrm{DE}}\right) \rho_{\mathrm{DE}}\right],
\end{aligned}
$$

i.e., it mimics the cosmology with $G_{\mathrm{cos}}=(1+\beta)^{2} G_{N}$ and the dark energy component.

\section{B. Cosmological perturbations}

We go on to the analysis of scalar perturbations around a cosmological background, with a particular focus on the case of a pressureless fluid. The perturbed ADM variables are given by

$$
N=\bar{N}(1+\delta n), \quad N_{i}=\bar{N} \partial_{i} \chi, \quad \gamma_{i j}=a^{2} e^{-2 \psi} \delta_{i j},
$$

where we used the spatial gauge degrees of freedom to write $\gamma_{i j}$ in the above form.

For the moment we work in the general action (3) to derive the quadratic action for the scalar perturbations, and later we will focus on the specific case of our interest. The action (3) can be expanded to quadratic order in perturbations as

$$
\begin{aligned}
S^{(2)}= & \int \mathrm{d} t \mathrm{~d}^{3} x \bar{N} a^{3}\left[-\frac{3 \beta_{0}}{\bar{\beta}_{1}}\left(\psi_{n}+\frac{H \delta n}{\bar{\beta}_{1}}\right)^{2}\right. \\
& -\frac{2 \beta_{0}}{\bar{\beta}_{1}}\left(\psi_{n}+\frac{H \delta n}{\bar{\beta}_{1}}\right) \frac{\Delta \chi}{a^{2}}+\frac{\beta_{0}\left(\bar{\beta}_{1}-\bar{\beta}_{2}\right)}{3 \bar{\beta}_{1} \bar{\beta}_{2}}\left(\frac{\Delta \chi}{a^{2}}\right)^{2} \\
& \left.-\left(\alpha_{2}+\frac{\alpha_{4}}{\bar{N}}\right) \frac{\psi \Delta \psi}{a^{2}}+2 \alpha_{2} \delta n \frac{\Delta \psi}{a^{2}}\right],
\end{aligned}
$$

where the notations $\psi_{n}:=\bar{N}^{-1} \partial_{t} \psi$ and $\bar{\beta}_{1,2}:=\beta_{1,2} / \bar{N}+$ 1 are used. The matter action (48) can also be expanded in terms of perturbations as

$$
\begin{aligned}
S_{\text {mat }}^{(2)}= & \int \mathrm{d} t \mathrm{~d}^{3} x \bar{N} a^{3}\left(\frac{\rho+P}{c_{s}^{2}}\right)\left[c_{s}^{2} \frac{\delta \varphi}{\varphi_{n}} \frac{\Delta \chi}{a^{2}}+\frac{\delta n^{2}}{2}\right. \\
& -\frac{\delta \varphi_{n}}{\varphi_{n}} \delta n-3 c_{s}^{2} \frac{\delta \varphi_{n}}{\varphi_{n}} \psi+\frac{1}{2}\left(\frac{\delta \varphi_{n}}{\varphi_{n}}\right)^{2} \\
& \left.+\frac{c_{s}^{2}}{2} \frac{\delta \varphi \Delta \delta \varphi}{\varphi_{n}^{2} a^{2}}\right]
\end{aligned}
$$

where $\delta \varphi$ is the perturbation of $\varphi$. Our total action is given by $S_{\text {tot }}^{(2)}=S^{(2)}+S_{\text {mat. }}^{(2)}{ }^{5}$ This is the complete expression for the quadratic action derived from the general action (3) in the presence of a perfect fluid (or a shiftsymmetric k-essence field).

Now we focus on the case of our interest: $\beta_{0}=\alpha_{2}=1$ and $\beta_{2}=\alpha_{4}=0$ with the notation $\beta_{1}=\beta$. For simplicity, we consider the background with $\bar{N}=1$. We follow the analysis of density perturbations in the extended cuscuton theory [29] to derive the reduced action written solely in terms of a single variable $\delta$ defined by

$$
\delta=\frac{\rho+P}{\rho c_{s}^{2}}\left(\frac{\dot{\delta \varphi}}{\dot{\varphi}}-\delta n\right)-\frac{3(\rho+P)}{\rho} \psi,
$$

where note that the density perturbation is given by $\delta \rho=$ $(\rho+P) / c_{s}^{2} \cdot(\dot{\delta} \dot{\varphi} / \dot{\varphi}-\delta n)$. Later at an appropriate point we will carefully take the limit $c_{s}^{2}, P \rightarrow 0$ while keeping $\rho$ finite. We start with introducing $\delta$ as an auxiliary field and write $S_{\text {tot }}^{(2)}$ equivalently as

$$
\begin{aligned}
S_{\mathrm{tot}}^{(2)}= & S^{(2)}+S_{\mathrm{mat}}^{(2)}-\int \mathrm{d} t \mathrm{~d}^{3} x a^{3}\left(\frac{\rho+P}{2 c_{s}^{2}}\right) \\
& \times\left[\frac{\dot{\delta \varphi}}{\dot{\varphi}}-\delta n-c_{s}^{2}\left(\frac{\rho \delta}{\rho+P}+3 \psi\right)\right]^{2} .
\end{aligned}
$$

The additional third term is introduced so that it removes $\dot{\delta} \varphi^{2}$ in $S_{\text {tot }}^{(2)}$. Namely, one ends up with the action that depends linearly on $\dot{\delta} \varphi$. The equation of motion for $\delta$ yields Eq. (76). Substituting this to Eq. (77), the third term vanishes and the action reduces back to the original one, $S^{(2)}+S_{\text {mat }}^{(2)}$. Thus, the two representations are indeed equivalent.

Since $S_{\text {tot }}^{(2)}$ is now linear in $\dot{\delta} \varphi$, one can use the equation of motion to remove $\delta \varphi$ from the action. At this stage one may substitute $P \propto Y^{(1+w) / 2 w}$ and express the action in terms of $\rho$ and $w$, which allows us for a $w \rightarrow 0$ limit without any divergences. Then, $\delta n$ and $\chi$ can also be

\footnotetext{
${ }^{5}$ We omit $\delta n \psi$ and $\psi^{2}$ from Eqs. (74) and (75) because upon combining $S^{(2)}$ and $S_{\text {mat }}^{(2)}$ they can be eliminated in the end by the use of the background equations.
} 
removed from the action by the use of their equations of motion. The resulting action is of the form

$$
\begin{aligned}
S_{\mathrm{tot}}^{(2)}= & \int \mathrm{d} t \mathrm{~d}^{3} x a^{3}\left[\dot{\delta} \mathcal{A}(t, \Delta) \dot{\delta}+\delta \mathcal{O}_{1}(t, \Delta) \delta\right. \\
& \left.+2 \psi \mathcal{O}_{2}(t, \Delta) \delta+\psi \mathcal{O}_{3}(t, \Delta) \psi\right],
\end{aligned}
$$

where

$$
\mathcal{A}=\frac{\rho}{3 \rho-2 \Delta / a^{2}} .
$$

The explicit expressions for $\mathcal{O}_{1}, \mathcal{O}_{2}$, and $\mathcal{O}_{3}$ are messy and not illuminating. Finally, one can eliminate $\psi$ by using its equation of motion and arrives at the reduced action expressed in terms of $\delta$ alone:

$$
S_{\mathrm{tot}}^{(2)}=\int \mathrm{d} t \mathrm{~d}^{3} x a^{3}[\dot{\delta} \mathcal{A}(t, \Delta) \dot{\delta}+\delta \mathcal{B}(t, \Delta) \delta] .
$$

On the basis of this action we move to study the behavior of the perturbations in the short and long wavelength limits. In both limits, we will see that the effect of modification of gravity comes into play only through the underlying background model.

\section{Short wavelength limit}

In the short wavelength limit, $\Delta / a^{2} \gg \rho$, we have

$$
\mathcal{A} \simeq-\frac{1}{2} a^{2} \rho \Delta^{-1}, \quad \mathcal{B} \simeq-\frac{1}{4} a^{2} \rho^{2} \Delta^{-1} .
$$

The time-dependent function in the action $\beta$ has dropped out. Since $\rho \propto a^{-3}$, the equation of motion for $\delta$ is found to be

$$
\ddot{\delta}+2 H \dot{\delta}=\frac{1}{2} \rho \delta .
$$

Recalling that $4 \pi G_{N}=1 /\left(2 \alpha_{2}\right)=1 / 2$, this is identical to the evolution equation for matter density perturbations in GR. Note, however, that the evolution of $\delta$ depends on the underlying background model, which could be different from that of the $\Lambda \mathrm{CDM}$ model. For example, if $G_{\mathrm{cos}} / G_{N} \neq 1$ as in the example in the previous subsection, the actual evolution of the density perturbations would be modified.

The equations of motion for $\delta n, \chi$, and $\psi$ allow us to write these variables in terms of $\delta$. In the short wavelength limit, we obtain

$$
\begin{aligned}
\delta n & \simeq \frac{1}{2} a^{2} \rho \Delta^{-1} \delta \\
\chi & \simeq-\frac{3 a^{4} \rho}{2(1+\beta)}\left[H \Delta^{-2} \delta+(1+\beta) \Delta^{-2} \dot{\delta}\right], \\
\psi & \simeq \frac{1}{2} a^{2} \rho \Delta^{-1} \delta .
\end{aligned}
$$

Therefore, the metric potentials that are used in the familiar Newtonian gauge analysis,

$$
\begin{aligned}
& \Phi:=\delta n+\dot{\chi}, \\
& \Psi:=\psi-H \chi,
\end{aligned}
$$

obey the standard relation,

$$
\Delta \Phi=\Delta \Psi=\frac{a^{2}}{2} \rho \delta,
$$

with $\delta \simeq \delta \rho / \rho$. Note that if one ignores the cosmic expansion, then this reproduces the result obtained in Sec. III.

\section{Long wavelength limit}

In the long wavelength limit, $\Delta / a^{2} \ll \rho$, we have

$$
\mathcal{A} \simeq \frac{1}{3}, \quad \mathcal{B} \simeq-\frac{1}{9} \frac{\Delta}{a^{2}} .
$$

Again, $\beta$-dependence has dropped out. The long wavelength solution to the equation of motion for $\delta$ is given by

$$
\delta \simeq \delta_{0}(\vec{x})-\frac{\Delta \delta_{0}(\vec{x})}{3} \int^{t} \frac{\mathrm{d} t^{\prime}}{a^{3}\left(t^{\prime}\right)} \int^{t^{\prime}} \mathrm{d} t^{\prime \prime} a\left(t^{\prime \prime}\right),
$$

where $\delta_{0}$ is independent of time and the decaying mode has been discarded. From the equations of motion for the other variables in the long wavelength limit, we obtain

$$
\begin{aligned}
\delta n & =\mathcal{O}\left(\Delta \delta / a^{2} H^{2}\right), \\
\chi & \simeq a^{2} \Delta^{-1} \dot{\delta}, \\
\psi & \simeq-\frac{\delta}{3},
\end{aligned}
$$

leading to

$$
\Phi=\Psi=-\frac{\delta_{0}(\vec{x})}{3}\left[1-\frac{H}{a} \int^{t} a\left(t^{\prime}\right) \mathrm{d} t^{\prime}\right] .
$$

The solution expressed in this way is the same as the long wavelength solution for $\Phi$ and $\Psi$ in the presence of a pressureless fluid and a cosmological constant in GR. In particular, in the matter-dominated era, $a \propto t^{2 / 3}$, we have the time-independent metric potentials, $\Phi=\Psi=-\delta_{0} / 5$. Note, however, that Eq. (94) has been derived without assuming any particular form of $a(t)$ for an accelerated phase of the cosmic expansion. In our modified theory of gravity, the background evolution (of the accelerated phase) could be different from that of the $\Lambda$ CDM model depending on the time-dependent functions in the action, so that the actual time-dependence of $\Phi$ and $\Psi$ could be different from that in the $\Lambda \mathrm{CDM}$ model away from the matter-dominated era. 


\section{WHEN MATTER MATTERS}

Finally, we discuss the problem of the coupling to generalized matter fields. Let us consider the cubic galileon [65] whose action is given by

$$
S_{\text {gal }}=\int \mathrm{d}^{4} x \sqrt{-g}\left[-\frac{1}{2}(\partial \varphi)^{2}-\frac{c}{2}(\partial \varphi)^{2} \square \varphi\right],
$$

where $c$ is a nonzero constant. In the following we will show that the extra dynamical scalar DOF would reappear when one adds to the action (3) the galileon as a matter field. This fact can be demonstrated by studying a quadratic action for scalar perturbations around a cosmological background.

Expanding the action (95) to second order in perturbations, we obtain

$$
\begin{aligned}
S_{\text {gal }}^{(2)}= & \int \mathrm{d} t \mathrm{~d}^{3} x \bar{N} a^{3}\left[\left(\varphi_{n}-3 c H \varphi_{n}^{2}\right) \delta \varphi \frac{\Delta \chi}{a^{2}}+c \varphi_{n}^{2} \delta \varphi_{n} \frac{\Delta \chi}{a^{2}}-c \varphi_{n}^{3} \delta n \frac{\Delta \chi}{a^{2}}-c \varphi_{n}^{2} \delta n \frac{\Delta \delta \varphi}{a^{2}}+\frac{\varphi_{n}^{2}}{2}\left(1-12 c H \varphi_{n}\right) \delta n^{2}\right. \\
& -\varphi_{n}\left(1-9 c H \varphi_{n}\right) \delta n \delta \varphi_{n}-3 c \varphi_{n}^{3} \delta n \psi_{n}-3\left(\varphi_{n}-3 c H \varphi_{n}^{2}\right) \psi \delta \varphi_{n}+3 c \varphi_{n}^{2} \psi_{n} \delta \varphi_{n}+\frac{1}{2}\left(1-6 c H \varphi_{n}\right) \delta \varphi_{n}^{2} \\
& \left.+\frac{1}{2 a^{2}}\left(1-4 c H \varphi_{n}-2 c \varphi_{n n}\right) \delta \varphi \Delta \delta \varphi\right],
\end{aligned}
$$

where $\delta \varphi$ is the fluctuation of the galileon field. We add this to the action (74): $S_{\text {tot }}^{(2)}=S^{(2)}+S_{\text {gal }}^{(2)}$. To highlight what causes the problem, we do not make any further simplification.

Using the equations of motion for $\delta n$ and $\chi$, one can eliminate them from the action. The resultant quadratic action is of the form

$$
S_{\mathrm{tot}}^{(2)}=\int \mathrm{d} t \mathrm{~d}^{3} x \bar{N} a^{3}\left(\mathcal{K}_{I J} q_{n}^{I} q_{n}^{J}+\cdots\right),
$$

where $q^{I}=\{\psi, \delta \varphi\}$ and we wrote only the terms quadratic in time derivatives. A straightforward calculation yields

$$
\operatorname{det} \mathcal{K}=-\frac{27\left[c \beta_{0}\left(\beta_{1} / \bar{N}\right) H \varphi_{n}^{2}\right]^{2}}{\Xi}
$$

with

$$
\begin{aligned}
\Xi:= & 12 \beta_{0}^{2} H^{2}-2 \beta_{0} \bar{\beta}_{1}^{2}\left(\bar{\beta}_{1}-\bar{\beta}_{2}\right) \varphi_{n}^{2} \\
& +12 c \beta_{0} \bar{\beta}_{1}\left[2 \bar{\beta}_{1}\left(\bar{\beta}_{1}-\bar{\beta}_{2}\right)+\bar{\beta}_{2}\right] H \varphi_{n}^{3} \\
& +3 c^{2} \bar{\beta}_{1}^{3} \bar{\beta}_{2} \varphi_{n}^{6} .
\end{aligned}
$$

This result shows that there are two scalar DOFs in the system unless $c \beta_{1}=0$; the scalar DOF reappears in the presence of a galileon field if it is minimally coupled to gravity in the frame where $\beta_{1} \neq 0$.

What we have seen here is analogous to the issue pointed out in the context of degenerate higher-order scalar-tensor theories $[66,67]$. In the case of the degenerate higher-order scalar-tensor theories, the constraint associated with the degeneracy is lost if a matter field is coupled to the Christoffel symbol. In the present case, the constraint that removes the scalar DOF in the gravity sector is lost due to the coupling of the cubic galileon to the Christoffel symbol.

\section{CONCLUSIONS AND OUTLOOK}

In this paper, we have studied aspects of spatially covariant theories of gravity with two tensorial degrees of freedom (DOFs), namely, modified gravity having the same number of DOFs as general relativity (GR). We have mainly focused on a subset of the general theories developed in Ref. [19] that is characterized by three time-dependent functions. Two of the three functions are related to the cuscuton terms [9] expressed in the unitary gauge, while the remaining one (denoted as $\beta$ ) is not included even in the extended cuscuton theory [12]. More specifically, we have worked with the action (8) with $\alpha_{2}=1$ and $\alpha_{4}=0$ as a particularly interesting subclass of the general action (3), with the purpose of exploring how the theory can be distinguished observationally or phenomenologically from GR.

First, we have seen that the theory of modified gravity under consideration can evade solar-system tests. We have also seen that the speed of gravitational waves is equal to the speed of light. Therefore, there is no obvious contradiction with observations and experiments at this point.

Next, we have studied black hole solutions ignoring the cuscuton terms that are supposed to be relevant only on cosmological scales. Since the only modification in the action appears in the coefficient of $K^{2}$, where $K$ is the trace of the extrinsic curvature of constant time hypersurfaces, the theory admits GR solutions foliated by maximal slices $(K=0)$. Therefore, we have the Schwarzschild solution. The situation here is essentially the same as that in Einstein-aether theory and the infrared limit of Hořava gravity. We have considered small deformations of the Schwarzschild solution, showing that no other asymptotically flat solutions can be obtained by a perturbative treatment. Our numerical analysis beyond the perturbative treatment supports the conclusion 
that the Schwarzschild solution is the only static, spherically symmetric, and asymptotically flat vacuum solution. We have also considered a slowly rotating black hole and obtained the Kerr solution in the slow-rotation approximation.

We have then investigated the cosmological dynamics of the homogeneous and isotropic background and scalar perturbations. Since we have a large degree of freedom to choose the time-dependent functions in the action including the cuscuton part, it is easy to realize the background evolution that is very close to or even identical to that of the $\Lambda$ CDM model in GR. As far as the long and short wavelength limits are concerned, we have found that the perturbation dynamics is modified only through the modification of the background evolution.

Combining these results, we conclude that it is quite difficult to distinguish modified gravity with the action (8) with $\alpha_{2}=1$ and $\alpha_{4}=0$ observationally or phenomenologically from GR.

Finally, we have noted, as a side remark, that in the presence of a galileon field in the matter sector, the dynamical scalar degree of freedom eliminated from the gravitational sector could reappear in a similar way to the case analyzed in Ref. [66, 67]. The result of our analysis implies that a galileon field must be coupled nonminimally to gravity in the frame where the action takes the form of Eq. (8).

Let us comment on several issues that are left for future work.

- Newtonian gravity is reproduced in the weak field regime, and black hole solutions in GR are obtained in the strong field regime in vacuo. It is then natural to move on to the study of the structure of relativistic stars.

- It would be interesting to study black hole perturbations and quasinormal modes, which could help us to distinguish modified gravity with two tensorial DOFs from GR.

- In the present paper, we have studied cosmological perturbations only in the long and short wavelength limits. On intermediate scales the perturbation evolution could differ from that in GR even if the background evolution coincides with that of the $\Lambda$ CDM model. A more detailed investigation is necessary.

- An application to inflationary cosmology would also be interesting.

These issues will be addressed in our forthcoming papers.

\section{ACKNOWLEDGMENTS}

The work of AI was supported by the JSPS Research Fellowships for Young Scientists No. 20J11285. The work of TK was supported by MEXT KAKENHI Grant Nos. JP20H04745 and JP20K03936.

\section{Appendix A: Equations of motion}

The equations of motion in vacuum derived from the action (8) are given as follows.

- The Hamiltonian constraint:

$$
K_{i j} K^{i j}-\frac{1}{3}\left[2\left(\frac{N}{\beta+N}\right)^{2}+1\right] K^{2}-\alpha_{1}-\alpha_{2} R=0 \text {. }
$$

- The momentum constraints:

$$
D_{j} \pi^{i j}=0
$$

where

$$
\pi^{i j}:=K^{i j}-\frac{1}{3}\left(\frac{2 N}{\beta+N}+1\right) K \gamma^{i j} .
$$

- The evolution equations:

$$
\begin{aligned}
& \frac{1}{N \sqrt{\gamma}} \partial_{t}\left(\sqrt{\gamma} \pi^{k l}\right) \gamma_{i k} \gamma_{j l}-\frac{1}{2}\left(\alpha_{1}+\frac{\alpha_{3}}{N}\right) \gamma_{i j} \\
& +2\left[K_{i k} K_{j}^{k}-\frac{1}{3}\left(\frac{2 N}{\beta+N}+1\right) K K_{i j}\right] \\
& -\frac{1}{2}\left[K_{k l} K^{k l}-\frac{1}{3}\left(\frac{2 N}{\beta+N}+1\right) K^{2}\right] \gamma_{i j} \\
& +\left(\alpha_{2}+\frac{\alpha_{4}}{N}\right)\left(R_{i j}-\frac{1}{2} R \gamma_{i j}\right) \\
& +\frac{\alpha_{2}}{N}\left(D^{2} N \gamma_{i j}-D_{i} D_{j} N\right) \\
& +\frac{1}{N}\left[D^{k}\left(\pi_{i k} N_{j}\right)+D^{k}\left(\pi_{j k} N_{i}\right)-D^{k}\left(\pi_{i j} N_{k}\right)\right]=0 .
\end{aligned}
$$

\section{Appendix B: Covariant form of the action}

In the main text, we use the ADM decomposition and consider the action of the form (3) which no longer has full diffeomorphism invariance. However, one can always restore it by introducing a Stückelberg field $\phi\left(x^{\mu}\right)$. In this appendix, we derive the covariant form of the action written in terms of the four-dimensional metric $g_{\mu \nu}$ and $\phi$.

The Stückelberg scalar field is introduced by writing the unit normal to constant time hypersurfaces as $n_{\mu}=$ $-\phi_{\mu} / \sqrt{2 X}$, where $\phi_{\mu}:=\nabla_{\mu} \phi$ and $X:=-\phi^{\mu} \phi_{\mu} / 2$ with $\nabla_{\mu}$ being the usual four-dimensional covariant derivative. The ADM variables in the action (3) are then replaced as follows:

$$
\begin{aligned}
N \rightarrow & \frac{1}{\sqrt{2 X}}, \\
K_{i j} \rightarrow \mathcal{K}_{\mu \nu}:= & -\frac{\phi_{\mu \nu}}{\sqrt{2 X}}+n_{\mu} a_{\nu}+n_{\nu} a_{\mu} \\
& +\frac{1}{2 X} n_{\mu} n_{\nu} n^{\rho} \nabla_{\rho} X \\
R \rightarrow{ }^{(4)} \mathcal{R}- & \mathcal{K}_{\mu \nu} \mathcal{K}^{\mu \nu}+\mathcal{K}^{2}-2 \nabla_{\mu}\left(\mathcal{K} n^{\mu}-a^{\mu}\right)
\end{aligned}
$$


where $\phi_{\mu \nu}:=\nabla_{\mu} \nabla_{\nu} \phi, a_{\mu}:=n^{\rho} \nabla_{\rho} n_{\mu}$, and ${ }^{(4)} \mathcal{R}$ is the four-dimensional Ricci scalar. One thus obtains

$$
\begin{aligned}
S= & \int \mathrm{d}^{4} x \sqrt{-g} \mathcal{L}, \\
\mathcal{L}= & {\left[\frac{1}{2} \widetilde{B}(\phi, X)-\widetilde{f}(\phi, X)\right]\left(\mathcal{K}_{\mu \nu} \mathcal{K}^{\mu \nu}-\mathcal{K}^{2}\right) } \\
& +\widetilde{C}(\phi, X) \mathcal{K}^{2}+\frac{1}{2}\left[\widetilde{\alpha}_{1}(\phi)+\widetilde{\alpha}_{3}(\phi) \sqrt{2 X}\right] \\
& +\widetilde{f}(\phi, X)\left[{ }^{(4)} \mathcal{R}-2 \nabla_{\mu}\left(\mathcal{K} n^{\mu}-a^{\mu}\right)\right]
\end{aligned}
$$

where

$$
\begin{aligned}
\widetilde{f} & =\frac{1}{2}\left[\widetilde{\alpha}_{2}(\phi)+\widetilde{\alpha}_{4}(\phi) \sqrt{2 X}\right], \\
\widetilde{B} & =\frac{\widetilde{\beta}_{0}(\phi)}{\widetilde{\beta}_{2}(\phi) \sqrt{2 X}+1}, \\
\widetilde{C} & =\frac{\widetilde{\beta}_{0}(\phi)}{3} \cdot \frac{\left[\widetilde{\beta}_{1}(\phi)-\widetilde{\beta}_{2}(\phi)\right] \sqrt{2 X}}{\left[\widetilde{\beta}_{1}(\phi) \sqrt{2 X}+1\right]\left[\widetilde{\beta}_{2}(\phi) \sqrt{2 X}+1\right]} .
\end{aligned}
$$

The time-dependent functions in the action (3) are given by $^{6}$

$$
\begin{aligned}
& \beta_{0}(t)=\widetilde{\beta}_{0}(\phi(t)), \quad \beta_{1,2}(t)=\dot{\phi}(t) \widetilde{\beta}_{1,2}(\phi(t)), \\
& \alpha_{1,2}(t)=\widetilde{\alpha}_{1,2}(\phi(t)), \quad \alpha_{3,4}(t)=\dot{\phi}(t) \widetilde{\alpha}_{3,4}(\phi(t)) .
\end{aligned}
$$

With some manipulation, the Lagrangian can be written in a more suggestive form as

$$
\begin{aligned}
\mathcal{L}= & \frac{\widetilde{\alpha}_{1}}{2}-\widetilde{\alpha}_{2}^{\prime \prime}(2 X-X \ln X)+\frac{\widetilde{\alpha}_{3}}{2} \sqrt{2 X}+\widetilde{\alpha}_{4}^{\prime \prime}(2 X)^{3 / 2} \\
& -\left(\frac{\widetilde{\alpha}_{2}^{\prime}}{2} \ln X+2 \widetilde{\alpha}_{4}^{\prime} \sqrt{2 X}\right) \square \phi+\widetilde{f}^{(4)} \mathcal{R}+\mathcal{L}_{\text {quad }},
\end{aligned}
$$

where a prime here denotes differentiation with respect to $\phi$ and

$$
\begin{aligned}
\mathcal{L}_{\text {quad }}= & A_{1} \phi_{\mu \nu} \phi^{\mu \nu}+A_{2}(\square \phi)^{2}+A_{3} \square \phi \phi^{\mu} \phi_{\mu \nu} \phi^{\nu} \\
& +A_{4} \phi^{\mu} \phi_{\mu \rho} \phi^{\rho \nu} \phi_{\nu}+A_{5}\left(\phi^{\mu} \phi_{\mu \nu} \phi^{\nu}\right)^{2},
\end{aligned}
$$

\footnotetext{
${ }^{6}$ The relation (B13) can be used to write the $\phi$-dependent functions explicitly, for example, in the first concrete model presented in Sec. VA (with $w=0$ ) as

$\widetilde{\alpha}_{1}=6\left\{\left[\frac{1}{\xi}-\frac{1}{(1+\beta)^{2}}\right] \phi^{2}-\frac{h_{0}^{2}}{\xi}\right\}$,

$\widetilde{\alpha}_{3}=\frac{4}{\phi^{2}-h_{0}^{2}}\left\{\left[\frac{1}{\xi}-\frac{1}{(1+\beta)^{2}}\right] \phi^{2}-\left(\frac{1}{\xi}-\frac{1}{1+\beta}\right) h_{0}^{2}\right\}$,

$\widetilde{\beta}_{1}=-\frac{2 \beta}{3\left(\phi^{2}-h_{0}^{2}\right)}$,

$\widetilde{\alpha}_{2}=\widetilde{\beta}_{0}=1, \quad \widetilde{\alpha}_{4}=\widetilde{\beta}_{2}=0$,
}

with the solution for the scalar field $\phi(t)=h_{0} \operatorname{coth}\left[(3 / 2) h_{0} t\right]$ with

$$
\begin{aligned}
& A_{1}=\frac{\widetilde{B}}{4 X}-\frac{\widetilde{f}}{2 X}, \quad A_{2}=-A_{1}+4 X^{2} A_{5}, \\
& A_{3}=-A_{4}+4 X A_{5}, \quad A_{4}=\frac{\widetilde{f}_{, X}+A_{1}}{X}, \\
& A_{5}=\frac{\widetilde{C}}{8 X^{3}} .
\end{aligned}
$$

This is a particular case of the U-degenerate theories, i.e., higher-order scalar-tensor theories that are degenerate when restricted to the unitary gauge [68]. It is easy to check that the above Lagrangian does not satisfy the degeneracy conditions in an arbitrary gauge if $\widetilde{C} \neq 0$. In general, there is one dynamical scalar DOF in a Udegenerate theory in the unitary gauge. However, with the above particular form of the Lagrangian, it turns out that $\phi$ does not propagate.

To demonstrate that $\phi$ does not propagate, let us study the equation of motion for $\phi$ in a Friedmann-LemaitreRobertson-Walker universe with the metric $\mathrm{d} s^{2}=-\mathrm{d} t^{2}+$ $a^{2}(t) \mathrm{d} \vec{x}^{2}$. For simplicity, we consider the case with $\alpha_{2}=$ $\beta_{0}=1$ and $\alpha_{4}=\beta_{2}=0$. The equation of motion for $\phi$ then reads

$$
\widetilde{\alpha}_{1}^{\prime} \dot{\phi}-3 H \widetilde{\alpha}_{3} \dot{\phi}-\frac{6 \widetilde{\beta}_{1} \dot{\phi} H}{\widetilde{\beta}_{1} \dot{\phi}+1} \mathcal{P}=0,
$$

where

$$
\mathcal{P}:=2 \frac{\mathrm{d}}{\mathrm{d} t}\left(\frac{H}{\widetilde{\beta}_{1} \dot{\phi}+1}\right)+\frac{3 H^{2}}{\widetilde{\beta}_{1} \dot{\phi}+1} .
$$

This equation does contain $\ddot{\phi}$ as well as $\dot{H}$. However, the ( $i j$ ) components of the field equations have the form,

$$
\mathcal{P}+\frac{1}{2}\left(\widetilde{\alpha}_{1}+\widetilde{\alpha}_{3} \dot{\phi}\right)+\cdots=0,
$$

where the ellipsis denotes the pressure of other matter fields. The evolution equations for the metric and $\phi$ are thus degenerate as in extended cuscuton theories [12], implying that the scalar DOF is in fact not dynamical.

Let us turn to discuss black hole solutions in the case where

$$
\begin{aligned}
& \widetilde{\beta}_{1}=\text { const }, \quad \widetilde{\beta}_{2}=0, \quad \widetilde{\alpha}_{1}=\alpha_{1}=\text { const } \\
& \widetilde{\alpha}_{2}=\alpha_{2}=\text { const }, \quad \widetilde{\alpha}_{3}=\widetilde{\alpha}_{4}=0 .
\end{aligned}
$$

Concerning black hole solutions, working in the covariant action results in much more involved equations. However, it is not difficult to see that the field equations admit the following configuration of the metric and $\phi$ :

$$
\begin{gathered}
\mathrm{d} s^{2}=-h(r) \mathrm{d} T^{2}+\frac{\mathrm{d} r^{2}}{h(r)}+r^{2} \mathrm{~d} \Omega^{2}, \\
\phi(T, r)=\frac{1}{N_{0}}\left(T+\int^{r} \frac{b_{0} / r^{2}}{h \sqrt{h+b_{0}^{2} / r^{4}}} \mathrm{~d} r\right),
\end{gathered}
$$


where

$$
h(r):=1+\frac{\alpha_{1}}{6 \alpha_{2}} r^{2}-\frac{\mu_{0}}{r}+\frac{1-\alpha_{2}}{\alpha_{2}} \frac{b_{0}^{2}}{r^{4}} .
$$

Note that $\phi$ is allowed to have the linear $T$ dependence thanks to the shift symmetry $\phi \rightarrow \phi+$ const. This is a so-called stealth solution (when $\alpha_{2}=1$ ), but, in contrast to the familiar examples in the literature, we have $X \neq$ const in the present case. (See Ref. [69] for a comprehensive discussion on stealth of solutions in quadratic degenerate higher-order scalar-tensor theories.) Moving to the unitary gauge, $\phi(T, r)=t$, this solution reproduces the one obtained in Sec. IV A.
[1] D. Lovelock, The Einstein tensor and its generalizations, J. Math. Phys. 12 (1971) 498.

[2] D. Lovelock, The four-dimensionality of space and the einstein tensor, J. Math. Phys. 13 (1972) 874.

[3] C. Cheung, P. Creminelli, A. L. Fitzpatrick, J. Kaplan and L. Senatore, The Effective Field Theory of Inflation, JHEP 03 (2008) 014 [0709.0293].

[4] G. Gubitosi, F. Piazza and F. Vernizzi, The Effective Field Theory of Dark Energy, JCAP 02 (2013) 032 [1210.0201].

[5] J. K. Bloomfield, E. E. Flanagan, M. Park and S. Watson, Dark energy or modified gravity? An effective field theory approach, JCAP 08 (2013) 010 [1211.7054].

[6] J. Gleyzes, D. Langlois and F. Vernizzi, A unifying description of dark energy, Int. J. Mod. Phys. D 23 (2015) 1443010 [1411.3712].

[7] X. Gao, Unifying framework for scalar-tensor theories of gravity, Phys. Rev. D 90 (2014) 081501 [1406.0822].

[8] X. Gao, Hamiltonian analysis of spatially covariant gravity, Phys. Rev. D 90 (2014) 104033 [1409.6708].

[9] N. Afshordi, D. J. H. Chung and G. Geshnizjani, Cuscuton: A Causal Field Theory with an Infinite Speed of Sound, Phys. Rev. D 75 (2007) 083513 [hep-th/0609150].

[10] G. W. Horndeski, Second-order scalar-tensor field equations in a four-dimensional space, Int. J. Theor. Phys. 10 (1974) 363.

[11] J. Gleyzes, D. Langlois, F. Piazza and F. Vernizzi, Healthy theories beyond Horndeski, Phys. Rev. Lett. 114 (2015) 211101 [1404.6495].

[12] A. Iyonaga, K. Takahashi and T. Kobayashi, Extended Cuscuton: Formulation, JCAP 12 (2018) 002 [1809.10935].

[13] C. Lin and S. Mukohyama, A Class of Minimally Modified Gravity Theories, JCAP 10 (2017) 033 [1708.03757].

[14] K. Aoki, C. Lin and S. Mukohyama, Novel matter coupling in general relativity via canonical transformation, Phys. Rev. D 98 (2018) 044022 [1804.03902].

[15] K. Aoki, A. De Felice, C. Lin, S. Mukohyama and M. Oliosi, Phenomenology in type-I minimally modified gravity, JCAP 01 (2019) 017 [1810.01047].

[16] A. De Felice, A. Doll and S. Mukohyama, A theory of type-II minimally modified gravity, JCAP 09 (2020) 034 [2004.12549].

[17] K. Aoki, M. A. Gorji and S. Mukohyama, A consistent theory of $D \rightarrow 4$ Einstein-Gauss-Bonnet gravity, Phys. Lett. B 810 (2020) 135843 [2005.03859].

[18] K. Aoki, F. Di Filippo and S. Mukohyama, Non-uniqueness of massless transverse-traceless graviton, JCAP 05 (2021) 071 [2103.15044].

[19] X. Gao and Z.-B. Yao, Spatially covariant gravity theories with two tensorial degrees of freedom: the formalism, Phys. Rev. D 101 (2020) 064018 [1910.13995].

[20] Y.-M. Hu and X. Gao, Spatially covariant gravity with two degrees of freedom: perturbative analysis, 2104.07615.

[21] P. Horava, Quantum Gravity at a Lifshitz Point, Phys. Rev. D 79 (2009) 084008 [0901.3775].

[22] N. Franchini, M. Herrero-Valea and E. Barausse, Relation between general relativity and a class of Horava gravity theories, Phys. Rev. D 103 (2021) 084012 [2103.00929].

[23] C. Deffayet, X. Gao, D. A. Steer and G. Zahariade, From k-essence to generalised Galileons, Phys. Rev. D 84 (2011) 064039 [1103.3260].

[24] T. Kobayashi, M. Yamaguchi and J. Yokoyama, Generalized G-inflation: Inflation with the most general second-order field equations, Prog. Theor. Phys. 126 (2011) 511 [1105.5723].

[25] X. Gao and Z.-B. Yao, Spatially covariant gravity with velocity of the lapse function: the Hamiltonian analysis, JCAP 05 (2019) 024 [1806.02811].

[26] D. Langlois and K. Noui, Degenerate higher derivative theories beyond Horndeski: evading the Ostrogradski instability, JCAP 02 (2016) 034 [1510.06930].

[27] M. Crisostomi, K. Koyama and G. Tasinato, Extended Scalar-Tensor Theories of Gravity, JCAP 04 (2016) 044 [1602.03119].

[28] J. Ben Achour, M. Crisostomi, K. Koyama, D. Langlois, K. Noui and G. Tasinato, Degenerate higher order scalar-tensor theories beyond Horndeski up to cubic order, JHEP 12 (2016) 100 [1608.08135].

[29] A. Iyonaga, K. Takahashi and T. Kobayashi, Extended Cuscuton as Dark Energy, JCAP 07 (2020) 004 [2003.01934].

[30] D. Glavan and C. Lin, Einstein-Gauss-Bonnet Gravity in Four-Dimensional Spacetime, Phys. Rev. Lett. 124 (2020) 081301 [1905.03601].

[31] H. Lu and Y. Pang, Horndeski gravity as $D \rightarrow 4$ limit of Gauss-Bonnet, Phys. Lett. B 809 (2020) 135717 [2003.11552].

[32] T. Kobayashi, Effective scalar-tensor description of regularized Lovelock gravity in four dimensions, JCAP 07 (2020) 013 [2003.12771].

[33] P. G. S. Fernandes, P. Carrilho, T. Clifton and D. J. Mulryne, Derivation of Regularized Field Equations for the Einstein-Gauss-Bonnet Theory in Four Dimensions, Phys. Rev. D 102 (2020) 024025 [2004.08362].

[34] R. A. Hennigar, D. Kubizñák, R. B. Mann and C. Pollack, On taking the $D \rightarrow 4$ limit of Gauss-Bonnet 
gravity: theory and solutions, JHEP 07 (2020) 027 [2004.09472].

[35] J. Bonifacio, K. Hinterbichler and L. A. Johnson, Amplitudes and $4 D$ Gauss-Bonnet Theory, Phys. Rev. D 102 (2020) 024029 [2004.10716].

[36] G. Tasinato, Symmetries for scalarless scalar theories, Phys. Rev. D 102 (2020) 084009 [2009.02157].

[37] J. C. Feng and S. Carloni, New class of generalized coupling theories, Phys. Rev. D 101 (2020) 064002 [1910.06978].

[38] S. Mukohyama and K. Noui, Minimally Modified Gravity: a Hamiltonian Construction, JCAP 07 (2019) 049 [1905.02000].

[39] Z.-B. Yao, M. Oliosi, X. Gao and S. Mukohyama, Minimally modified gravity with an auxiliary constraint: A Hamiltonian construction, Phys. Rev. D 103 (2021) 024032 [2011.00805].

[40] K. Aoki, A. De Felice, S. Mukohyama, K. Noui, M. Oliosi and M. C. Pookkillath, Minimally modified gravity fitting Planck data better than $\Lambda$ CDM, Eur. Phys. J. C 80 (2020) 708 [2005.13972].

[41] A. De Felice and S. Mukohyama, Weakening gravity for dark matter in a type-II minimally modified gravity, JCAP 04 (2021) 018 [2011.04188].

[42] J. Sangtawee and K. Karwan, Inflationary model in minimally modified gravity theories, Phys. Rev. D 104 (2021) 023511 [2103.11463].

[43] J. D. Bekenstein, The Relation between physical and gravitational geometry, Phys. Rev. D 48 (1993) 3641 [gr-qc/9211017].

[44] D. Blas, O. Pujolas and S. Sibiryakov, Consistent Extension of Horava Gravity, Phys. Rev. Lett. 104 (2010) 181302 [0909.3525].

[45] D. Blas, O. Pujolas and S. Sibiryakov, Models of non-relativistic quantum gravity: The Good, the bad and the healthy, JHEP 04 (2011) 018 [1007.3503].

[46] T. Jacobson and D. Mattingly, Gravity with a dynamical preferred frame, Phys. Rev. D 64 (2001) 024028 [gr-qc/0007031].

[47] D. Blas, O. Pujolas and S. Sibiryakov, Comment on 'Strong coupling in extended Horava-Lifshitz gravity', Phys. Lett. B 688 (2010) 350 [0912.0550].

[48] T. Jacobson, Extended Horava gravity and Einstein-aether theory, Phys. Rev. D 81 (2010) 101502 [1001.4823].

[49] M. Li and Y. Pang, A Trouble with Hořava-Lifshitz Gravity, JHEP 08 (2009) 015 [0905.2751].

[50] M. Henneaux, A. Kleinschmidt and G. Lucena Gómez, A dynamical inconsistency of Horava gravity, Phys. Rev. D 81 (2010) 064002 [0912.0399].

[51] C. M. Will, Theory and Experiment in Gravitational Physics. Cambridge University Press, 2018.

[52] LiGO Scientific, Virgo collaboration, B. P. Abbott et al., GW170817: Observation of Gravitational Waves from a Binary Neutron Star Inspiral, Phys. Rev. Lett. 119 (2017) 161101 [1710.05832].
[53] LigO Scientific, Virgo, Fermi-GBM, INTEGRAL collaboration, B. P. Abbott et al., Gravitational Waves and Gamma-rays from a Binary Neutron Star Merger: GW170817 and GRB 170817A, Astrophys. J. Lett. 848 (2017) L13 [1710.05834].

[54] E. Barausse, T. Jacobson and T. P. Sotiriou, Black holes in Einstein-aether and Horava-Lifshitz gravity, Phys. Rev. D 83 (2011) 124043 [1104.2889].

[55] D. Blas and S. Sibiryakov, Horava gravity versus thermodynamics: The Black hole case, Phys. Rev. D 84 (2011) 124043 [1110.2195].

[56] P. Berglund, J. Bhattacharyya and D. Mattingly, Mechanics of universal horizons, Phys. Rev. D 85 (2012) 124019 [1202.4497].

[57] A. De Felice, A. Doll, F. Larrouturou and S. Mukohyama, Black holes in a type-II minimally modified gravity, JCAP 03 (2021) 004 [2010.13067].

[58] K. Lin, S. Mukohyama, A. Wang and T. Zhu, No static black hole hairs in gravitational theories with broken Lorentz invariance, Phys. Rev. D 95 (2017) 124053 [1704.02990].

[59] E. Barausse and T. P. Sotiriou, Black holes in Lorentz-violating gravity theories, Class. Quant. Grav. 30 (2013) 244010 [1307.3359].

[60] E. Barausse, T. P. Sotiriou and I. Vega, Slowly rotating black holes in Einstein-ather theory, Phys. Rev. D 93 (2016) 044044 [1512.05894].

[61] A. Adam, P. Figueras, T. Jacobson and T. Wiseman, Rotating black holes in Einstein-aether theory, 2108.00005.

[62] J. B. Hartle, Slowly rotating relativistic stars. 1. Equations of structure, Astrophys. J. 150 (1967) 1005.

[63] J. B. Hartle and K. S. Thorne, Slowly Rotating Relativistic Stars. II. Models for Neutron Stars and Supermassive Stars, Astrophys. J. 153 (1968) 807.

[64] S. M. Carroll and E. A. Lim, Lorentz-violating vector fields slow the universe down, Phys. Rev. D 70 (2004) 123525 [hep-th/0407149].

[65] A. Nicolis, R. Rattazzi and E. Trincherini, The Galileon as a local modification of gravity, Phys. Rev. D 79 (2009) 064036 [0811.2197].

[66] C. Deffayet and S. Garcia-Saenz, Degeneracy, matter coupling, and disformal transformations in scalar-tensor theories, Phys. Rev. D 102 (2020) 064037 [2004.11619].

[67] S. Garcia-Saenz, On the Coupling of Generalized Proca Fields to Degenerate Scalar-Tensor Theories, Universe 7 (2021) 190 [2106.14960].

[68] A. De Felice, D. Langlois, S. Mukohyama, K. Noui and A. Wang, Generalized instantaneous modes in higher-order scalar-tensor theories, Phys. Rev. D 98 (2018) 084024 [1803.06241].

[69] K. Takahashi and H. Motohashi, General Relativity solutions with stealth scalar hair in quadratic higher-order scalar-tensor theories, JCAP 06 (2020) 034 [2004.03883]. 